\title{
Temporal similarity for optical short pulses
}

\author{
J. Ojeda-Castañeda, ${ }^{*}$ and C. M. Gómez-Sarabia \\ Electronics Department, University of Guanajuato, Salamanca, 36885, Guanajuato, México
}

Received November 29, 2010; accepted December 14, 2010; published December 31, 2010

\begin{abstract}
We define a temporal similarity function for assessing the temporal evolution of short pulses in dispersion media. For first-order dispersion media the temporal similarity function has a simple close expression. We use the sampled values of the temporal similarity function as coefficients, for expressing the output complex amplitude envelope as a finite linear superposition of the input complex amplitude envelope.
\end{abstract}

For analyzing the propagation of modes in optical fibres, it is convenient to compare the near field diffraction patterns against the far field diffraction pattern. One can do a quantitative comparison of similarity, by performing a spatial cross-correlation between near field diffraction patterns and the far field diffraction pattern [1]. For a quantitative comparison between an initial complex amplitude distribution and its Fresnel diffraction pattern, Lohmann and OjedaCastañeda used a cross-correlation between an initial complex amplitude distribution and its Fresnel diffraction pattern [2]. This latter cross-correlation is useful for setting array illuminators $[3,5]$.

Our aim here is to define and to explore the use of a temporal similarity function, which is a temporal crosscorrelation between the input complex amplitude envelope and the output complex amplitude envelope, of the same dispersive medium. If the input is represented by a Dirac comb, then at discrete values the temporal similarity function has simple mathematical expressions. We show that by using the sampled values of the temporal similarity function, as coefficients, one can express the output complex amplitude envelope as a finite linear superposition of the input complex amplitude envelope.

\section{Temporal similarity}

If one neglects nonlinear effects, inside a dispersive medium, one can express the evolution of the slowly varying complex envelope as follows:

$u(\tau, z)=(1 / 2 \pi) \int_{-\infty}^{\infty} U(\omega) H\left(\omega ; z ; \beta_{m}\right) \exp (-i \tau \omega) d \omega$.
In Eq. (1) $\tau$ is the time measured in the proper reference frame of the pulse; we denote as $U(\omega)$ the Fourier spectrum of the input complex amplitude envelope; and $\mathrm{H}\left(\omega ; \mathrm{z} ; \beta_{\mathrm{m}}\right)$ represents the transfer function at a distance $\mathrm{z}$, inside the dispersive medium. In a symbolic fashion, the letter $\beta_{\mathrm{m}}$ indicates a dispersion coefficient, in the Taylor series expansion of the dispersion relation around $\omega_{0}$. Trivially, if $z=0$ then $\mathrm{H}\left(\omega ; 0 ; \beta_{\mathrm{m}}\right)=1$, Eq. (1) reduces to a Fourier transform, which relates the input complex amplitude envelope with its Fourier spectrum. For a firstorder dispersion medium, with dispersion coefficient $\beta_{2}$, Eq. (1) becomes

$$
u(\tau, z)=(1 / 2 \pi) \int_{-\infty}^{\infty} U(\omega) \exp \left(-i z\left(\beta_{2} / 2\right) \omega^{2}\right) \exp (i \tau \omega) d \omega .
$$

And in a similar fashion, for a second-order dispersion medium, with dispersion coefficient $\beta_{3}$, Eq. (1) becomes

$$
u(\tau, z)=(1 / 2 \pi) \int_{-\infty}^{\infty} U(\omega) \exp \left(-i z\left(\beta_{3} / 6\right) \omega^{3}\right) \exp (i \tau \omega) d \omega .
$$

Next, we use the proposal in Ref. [2] for defining the following temporal, cross-correlation function

$$
S(t ; z)=\int_{-\infty}^{\infty} u(\tau+t, z) u^{*}(\tau, 0) d \tau
$$

If one substitutes Eq. (1), at $z \neq 0$ and at $z=0$ in Eq. (4), we obtain the following result

$$
S(t ; z)=(1 / 2 \pi) \int_{-\infty}^{\infty}|U(\omega)|^{2} H\left(\omega ; \beta_{m} ; z\right) \exp (\text { it } \omega) d \omega .
$$

If the input complex envelope is periodic, then

$$
|U(\omega)|^{2}=(2 \pi) \sum_{q=-\infty}^{\infty}\left|a_{q}\right|^{2} \delta(\omega-q \Omega) .
$$

*E-mail: jorge_ojedacastaneda@yahoo.com 
And hence, by substituting Eq. (6) in Eq. (5), we obtain that for periodic short pulses the temporal similarity function is

$$
S(t ; z)=\left(\sum_{q=-\infty}^{\infty}\left|a_{q}\right|^{2} H\left(q \Omega ; \beta_{m} ; z\right) \exp (i q \Omega t) .\right.
$$

It is apparent from Eq. (7) that the temporal similarity function is periodic in time. Next, we recognize that the temporal similarity function can also be a periodic function along the z-axis. For a first-order dispersion medium, Eq. (7) becomes

$$
S(t ; z)=\sum_{q=-\infty}^{\infty}\left|a_{q}\right|^{2} \exp \left[-i 2 \pi z\left(\Omega^{2} \beta_{2} / 4 \pi\right) q^{2}\right] \exp (i q \Omega t) .
$$

And for a second-order dispersion medium, Eq. (7) takes the form

$$
S(t ; z)=\sum_{q=-\infty}^{\infty}\left|a_{q}\right|^{2} \exp \left[-i 2 \pi z\left(\Omega^{3} \beta_{3} / 12 \pi\right) q^{3}\right] \exp (i q \Omega) .
$$

In both cases the period along the $\mathrm{z}$-axis, $\mathrm{Z}_{\mathrm{T}}$, is the Talbot length in the respective dispersion medium. That is, for a first-order dispersion medium, with $\beta_{2}>0$,

$$
Z_{T}=4 \pi /\left(\Omega^{2} \beta_{2}\right)
$$

And for a second-order dispersion medium, with $\beta_{3}>0$,

$$
Z_{T}=12 \pi /\left(\Omega^{3} \beta_{3}\right)
$$

Next, we illustrate our proposal with simple applications.

\section{Fractional Talbot effect}

For gaining a physical insight into the above definition and its mathematical expressions, we assume that at the input we have a Dirac comb. For this extreme case, the properties of the temporal similarity function are closely related to the complex amplitude envelope itself. In next section, we clarify the importance of evaluating temporal similarity function at discrete values of $\mathrm{t}=(\mathrm{n} / \mathrm{N}) \mathrm{T}$. If the input is Dirac comb, then Eq. (7) becomes

$$
S(t ; z)=\sum_{q=-\infty}^{\infty} H\left(q \Omega ; \beta_{m} ; z\right) \exp (i q \Omega t)
$$

And if $\mathrm{t}=(\mathrm{n} / \mathrm{N}) \mathrm{T}$, then Eq. (11) can be written as a discrete Fourier transform:
$S((n / N) T ; z)=\sum_{q=-\infty}^{\infty} H\left(q \Omega ; \beta_{m} ; z\right) \exp (i 2 \pi n q / N)$.

Hence, the discrete version of the temporal similarity function can be thought of as a discrete impulse response [6]. Next, we note that at a distance $\mathrm{z}=\mathrm{Z}_{\mathrm{T}} / \mathrm{N}$, for a firstorder dispersion medium, the discrete version of Eq. (8) becomes

$$
S\left(n T / N ; Z_{T} / N\right)=\sum_{q=-\infty}^{\infty} \exp \left(-i 2 \pi q^{2} / N\right) \exp (i 2 \pi n q / N) .
$$

And for the second-order dispersion medium, the discrete version of Eq. (9) becomes

$$
S\left(n T / N ; Z_{T} / N\right)=\sum_{q=-\infty}^{\infty} \exp \left(-i 2 \pi q^{3} / N\right) \exp (-i 2 \pi n q / N)
$$

For describing Fresnel diffraction, Guigay [7] reported a closed formula for evaluating equation (13):

$$
\begin{aligned}
S\left(n T / N ; Z_{T} / N\right)= & \lfloor\exp (-i \pi / 4) / \sqrt{2 N}\rfloor \\
& {\left[1+(-1)^{n}(i)^{N}\right] \exp \left(-i \pi n^{2} / 2 N\right) . }
\end{aligned}
$$

As far as we know, there is no analytical formula for evaluating Eq. (14). In Table 1 we display the values of the square modulus of Eq. (15). That is,

$$
\left|S\left(n T / N ; Z_{T}\right)\right|^{2}=(1 / N)\left[1+(-1)^{n} \cos (\pi N / 2)\right]
$$

\begin{tabular}{|c|c|c|}
\hline $\mathrm{N}$ & If $\mathrm{n}=2 \mathrm{q}$ & If $\mathrm{n}=2 \mathrm{q}+1$ \\
\hline $2,6,10, \ldots$ & 0 & $(2 / \mathrm{N})$ \\
\hline $4,8,12, \ldots$ & $(2 / \mathrm{N})$ & 0 \\
\hline $3,5,7, \ldots$ & $(1 / \mathrm{N})$ & $(1 / \mathrm{N})$ \\
\hline
\end{tabular}

Table 1. Square modulus of the temporal similarity function.

Along column one, in Table 1, we list three family sets of values of $\mathrm{N}$. Along column two, we specify the values of $\left|\mathrm{S}\left(\mathrm{nT} / \mathrm{N} ; \mathrm{Z}_{\mathrm{T}}\right)\right|^{2}$ if the index $\mathrm{n}$ is an integer, even number; while along column three, the index $\mathrm{n}$ is an integer, odd number. It is apparent from Table 1 that if $\mathrm{N}$ is an odd integer number, then $\left|\mathrm{S}\left(\mathrm{nT} / \mathrm{N} ; \mathrm{Z}_{\mathrm{T}}\right)\right|^{2}$ has a fixed value for 
any $\mathrm{n}=0, \ldots(\mathrm{N}-1)$. This result is agreement to the fact that the output complex amplitude envelope exhibits $(\mathrm{N}$ 1) interlaced spikes, between two consecutive deltas of the initial Dirac comb. Hence, at $\mathrm{z}=\mathrm{Z}_{\mathrm{T}} / \mathrm{N}$, the repetition rate increases by a factor $\mathrm{N}$. However, an increment in the repetion rate is associated to a power spectrum reduction by a factor $(1 / N)$. The result in Eq. (16) suggests the use of phase-only modulation, over the initial Dirac comb, for setting temporal Talbot array illuminators [6].

\section{Quasi-Gabor expansion}

Here we clarify the relevance of sampling the temporal similarity function. To that end, we use a Dirac comb for defining a family set of $\mathrm{N}$ periodic functions, with period $\mathrm{T}$. The $\mathrm{n}$-th member of the family set is

$$
\phi_{n}(\tau)=\sum_{q=-\infty}^{\infty} \delta(\tau-q T-n T / N)
$$

In equation (17) $\mathrm{N}$ is an arbitrary positive integer number; and $\mathrm{n}=0,1 \ldots(\mathrm{N}-1)$; and $\phi_{n}(\tau)=\phi_{0}(\tau-n T / N)$. It is straightforward to show that the members (of this family of functions) form an orthonormal set. That is,

$$
\delta_{m, n}=\int_{-T / 2}^{T / 2} \phi_{m}(\tau) \phi_{n}(\tau) d \tau
$$

In heuristic terms, if one keeps in mind Simpson's rule for integration, then one can assume that for large values of $\mathrm{N}$, the above family set is a complete set. Hence, we propose to use the following expansion

$$
u(\tau, z)=\sum_{n=0}^{N-1} C_{n}\left(z ; \beta_{m}\right) \phi_{0}(\tau-n T / N) .
$$

In Eq. (19) we denote the influence of the dispersion coefficient by using the letter $\beta_{\mathrm{m}}$, as a parameter. The finite linear superposition, in Eq. (19), can be considered as a quasi Gabor expansion [8]. The coefficients $\mathrm{C}_{\mathrm{n}}(\mathrm{z}$; $\beta_{\mathrm{m}}$ ) are obtained by evaluating the integral

$$
\begin{aligned}
C_{n}\left(z ; \beta_{m}\right) & =\int_{-T / 2}^{T / 2} u(\tau ; z) \phi_{0}(\tau-n T / N) d \tau \\
& =\int_{-T / 2}^{T / 2} u(\tau+n T / N ; z) \phi_{0}(\tau) d \tau .
\end{aligned}
$$

If the input complex amplitude envelope is $u(\tau, 0)=\phi_{0}(\tau)$, then the temporal similarity function in Eq. (4) is

$$
S(t ; z)=\int_{-T / 2}^{T / 2} u(\tau+t, z) \phi_{0}(\tau) d \tau .
$$

By comparison of Eq. (20) and (21) we claim the following. If at the input the complex amplitude envelope is a Dirac comb, then at a discrete set of values, $\mathrm{t}=(\mathrm{n} / \mathrm{N}) \mathrm{T}$, the temporal similarity function is the coefficient of a quasi Gabor expansion. In other words, the impulse response in Eq. (8) and (21) is

$$
S(n T / N ; z)=C_{n}\left(z ; \beta_{m}\right)
$$

The result in Eq. (22) offers a useful interpretation to a quasi-Gabor expansion. The coefficients are measurements of similarity between the input complex amplitude envelope and the output complex amplitude envelope, at the same dispersive medium.

We have defined a temporal similarity function, as a temporal cross-correlation between the input complex amplitude envelope, and the output complex amplitude envelope, of the same dispersive medium. For periodic short pulses the temporal similarity function has simple expressions, which shows that the temporal similarity function is periodic both in time, and along the $\mathrm{z}$-axis. The period along the $\mathrm{z}$-axis, $\mathrm{Z}_{\mathrm{T}}$, is the Talbot length in the respective dispersion medium.

For a first-order dispersion medium, the discrete version of the temporal similarity function follows the close expression reported by Guigay. We have shown that by using the sampled values of the temporal similarity function, as coefficients, one can express the output complex amplitude envelope as a finite linear superposition of the input complex amplitude envelope. The coefficients measure the similarity between the input complex amplitude envelope and the output complex amplitude envelope.

We are indebted to Adolf W. Lohmann, Pedro Andres, Jesus Lancis and Omel Mendoza-Yero for useful discussions on this topic.

\section{References}

[1] M. D. Feit and J. A. Fleck, Appl. Opt. 18, 2843 (1979).

[2] A.W. Lohmann and J. Ojeda-Castañeda, Opt. Comm. 249, 397 (2005).

[3] A.W. Lohmann, Optik 79, 41 (1988).

[4] J. R. Leger and G.J. Swanson, Opt. Lett. 15, 288 (1990)

[5] V. Arrizón and J. Ojeda-Castañeda, Appl. Opt. 33, 5925 (1994).

[6] J. Ojeda-Castañeda, P. Andrés and O. Mendoza-Yero, Optical Memory and Neural Networks 18, 260 (2009).

[7] J. P. Guigay, Optica Acta 18, 677 (1971).

[8] M. J. Bastiaans, Signal and Image Representation in Combined Spaces (Academic Press, 1995). 